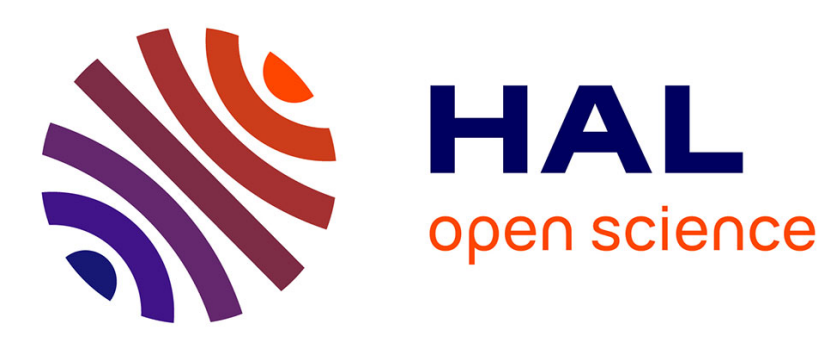

\title{
A Convex-Analysis Perspective on Disjunctive Cuts
}

Gérard Cornuéjols, Claude Lemaréchal

\section{To cite this version:}

Gérard Cornuéjols, Claude Lemaréchal. A Convex-Analysis Perspective on Disjunctive Cuts. [Research Report] RR-5317, INRIA. 2004, pp.17. inria-00070683

\section{HAL Id: inria-00070683 https://hal.inria.fr/inria-00070683}

Submitted on 19 May 2006

HAL is a multi-disciplinary open access archive for the deposit and dissemination of scientific research documents, whether they are published or not. The documents may come from teaching and research institutions in France or abroad, or from public or private research centers.
L'archive ouverte pluridisciplinaire HAL, est destinée au dépôt et à la diffusion de documents scientifiques de niveau recherche, publiés ou non, émanant des établissements d'enseignement et de recherche français ou étrangers, des laboratoires publics ou privés. 
INSTITUT NATIONAL DE RECHERCHE EN INFORMATIQUE ET EN AUTOMATIQUE

\section{A Convex-Analysis Perspective on Disjunctive Cuts}

Gérard Cornuéjols - Claude Lemaréchal

\section{$\mathbf{N}^{\circ} \mathbf{5 3 1 7}$}

Septembre 2004

THÈME 4 



\title{
A Convex-Analysis Perspective on Disjunctive Cuts *
}

\author{
Gérard Cornuéjols ${ }^{\dagger}$, Claude Lemaréchal ${ }^{\ddagger}$ \\ Thème 4 - Simulation et optimisation \\ de systèmes complexes \\ Projet Bipop \\ Rapport de recherche $n^{\circ} 5317$ - Septembre 2004 - 17 pages
}

\begin{abstract}
We treat the general problem of cutting planes with tools from convex analysis. We emphasize the case of disjunctive polyhedra and the generation of facets. We conclude with some considerations on the design of disjunctive cut generators.
\end{abstract}

AMS Subject Classification (2000) 52A41, $90 \mathrm{C} 11$

Key-words: Integer programming, cutting planes, separation, disjunctive cut, lift and project, convex analysis

* Supported by NSF grant DMII-0352885, ONR grant N00014-97-1-0196, INRIA grant ODW and IBM.

$\dagger$ Tepper School of Business, Carnegie Mellon University, Pittsburgh, PA, 15213; and LIF, Faculté des Sciences de Luminy, 13288 Marseille, France. gc0v@andrew.cmu.edu

$\ddagger$ Inria, 655 avenue de l’Europe, Montbonnot, 38334 Saint Ismier, France. claude.lemarechal@inrialpes .fr 


\section{Un Point de Vue d'Analyse Convexe sur les Coupes Disjonctives}

Résumé : Nous considérons sous l'angle de l'analyse convex le probème général des plans sécants en optimisation combinatoire. Nous nous intéressons particulièrement aux coupes disjonctives et à la construction de facettes. Nous terminons par quelques considérations sur le développement de générateurs de coupes efficaces.

Mots-clés : Programmation en nombres entiers, plans sécants, séparation, lift and project, coupe disjonctive, anaylse convexe 


\section{Introduction}

An important tool in combinatorial optimization is cut generation:

(i) a linear function $c^{\top} x$ must be maximized over a "hard-to-deal-with" set $S \subset \mathbb{R}^{n}$ (typically a huge set of integer points);

(ii) for want of a tractable method, $S$ is relaxed to some "easier" set $R$, usually polyhedral, over which $c^{\top} x$ is maximized;

(iii) this may produce some $\bar{x}$ not even belonging to $\overline{\mathrm{conv}}(S)$, the closed convex hull of $S$;

(iv) then the problem is to separate $\bar{x}$ from $S$ by some cutting plane(s), in order to describe $S-$ or $\overline{\operatorname{conv}}(S)$ more accurately.

A cutting plane is defined by the equation $d^{\top} x=\kappa$, where the nonzero direction $d \in \mathbb{R}^{n}$ and the righthand side $\kappa \in \mathbb{R}$ satisfy

$$
d^{\top} \bar{x}>\kappa \quad \text { and } \quad d^{\top} x \leqslant \kappa \text { for all } x \in S
$$

or equivalently: $d^{\top} \bar{x}>\sigma_{S}(d)$, where $\sigma_{S}$ is the so-called support function of $S$ (some essential concepts from convex analysis are given in the Appendix).

Because $S$ is hard to deal with directly, a possible approach for constructing cutting planes is to introduce an intermediate set $P \supset S$ not containing $\bar{x}$ and to find $d$ separating $\bar{x}$ from $P$. Some desirable properties for a good cut are as follows:

- It should touch $P$, which fixes $\kappa$ to its smallest possible value: $\kappa=\sigma_{P}(d)$. Thus, finding a cut is a problem in $d \in \mathbb{R}^{n}$ only.

- It should be as deep as possible, i.e. $d^{\top} \bar{x}-\sigma_{P}(d)$ should be maximal in a certain sense. Naturally, this requires in particular an appropriate normalization of $d$ (to avoid obtaining $d^{\top} \bar{x}-\sigma_{P}(d)=+\infty$ ); several works are devoted to defining suitable normalizations $[2,6,3,11]$.

- When the enlargement $P$ is polyhedral, a good cut should also touch $P$ on a facet; this means that the face $\mathrm{F}_{P}(d)$ of $P$ exposed by $d$ (see (15) in the Appendix) should have maximal dimension.

The present paper is thus devoted to the general problem of defining good cuts according to the above criteria; and for this we use the language and tools from convex analysis.

An application that we have in mind is in integer programming. For example in the case of pure 0-1 programming, $S$ has the form $S:=R \cap\{0,1\}^{n}$ for some given polyhedron $R \subset[0,1]^{n}$. Optimizing $c^{\top} x$ over the relaxation $R$ yields some point $\bar{x}$. When $\bar{x} \in\{0,1\}^{n}$, the integer program is solved. When this is not the case, then $0<\bar{x}_{j}<1$ for some $j$, and a natural choice for the above-mentioned enlargement $P$ of $S$ is the (convex hull of the) union of the polyhedra

$$
R \cap\left\{x: x_{j}=0\right\} \text { and } R \cap\left\{x: x_{j}=1\right\} .
$$

The inequalities that separate $\bar{x}$ from this set $P$ are called the lift-and-project cuts [2]. In practice, a small set of lift-and-project cuts with the good characteristics mentioned earlier are appended to the definition of $R$, thus strengthening the relaxation. The process can be repeated several times for further strengthening.

In a mixed integer linear program, $S:=R \cap\left(\mathbb{N}^{p} \times \mathbb{R}^{q}\right)$ where $p, q$ are positive integers such that $p+q=n$, and $R \subset \mathbb{R}^{n}$ is a polyhedron. If the point $\bar{x}$ obtained by optimizing $c^{\top} x$ over $R$ does not belong to $\overline{\text { conv }}(S)$, one may strengthen the relaxation $R$ by adding cuts, such as split cuts [8] defined as follows. Consider a disjunction " $\pi^{\top} x \leqslant \pi_{0}$ or $\pi^{\top} x \geqslant \pi_{0}+1$ " satisfied by all points of $S$ but not by $\bar{x}$. A particular choice that gives a valid disjunction for $S$ is $\left(\pi, \pi_{0}\right) \in \mathbb{N}^{n+1}$ with $\pi_{j}=0$ for $j=p+1, \ldots, n$. The set $P$ is the union of

$$
R \cap\left\{x: \pi^{\top} x \leq \pi_{0}\right\} \text { and } R \cap\left\{x: \pi^{\top} x \geq \pi_{0}+1\right\}
$$

and split cuts are inequalities that separate $\bar{x}$ from $P$. A lift-and-project cut is a special type of split cut. Another well-known example is Gomory's mixed integer cut [9].

More generally, disjunctive programming, as was introduced by Balas in the 1970's [1], considers a union of polyhedra. Specifically, given a finite number of polyhedra $P_{i}$, one would like to separate $\bar{x}$ from $\cup_{i} P_{i}$ or equivalently from $P:=\overline{\mathrm{conv}}\left(\cup_{i=1}^{k} P_{i}\right)$. We will refer to $P$ as the disjunctive polyhedron. Unfortunately, a description of the disjunctive polyhedron as $P=\left\{x \in \mathbb{R}^{n}: A x \leqslant b\right\}$ may require an exponential number of constraints $A x \leqslant b$. Balas' key result is that $P$ has a compact representation in a higher-dimensional space, namely there is a polyhedron $\tilde{P}:=\left\{(x, y) \in \mathbb{R}^{n+p}: C x+D y \leqslant d\right\}$, whose projection onto the $x$-space is $P$, and $\tilde{P}$ has only around $k n$ variables and $\sum_{i=1}^{k} m_{i}$ constraints; here $k$ is the number of polyhedra and $m_{i}$ denotes the number of constraints of $P_{i}$. As a consequence, separating $\bar{x}$ from $P$ can be solved efficiently by linear programming. We also mention [7], in which unions of non-polyhedral sets are considered. 
The paper is organized as follows. Sections 2 to 4 introduce from different points of view the fundamental objects for cutting: the reverse polar cone and its associated support function. In $\S 5$ they are particularized to unions of sets and the polyhedral case is studied in $\S 6$. Finally we have a conclusion section: it turns out that good cuts between $\bar{x}$ and $P$ are attached to the direction pointing from $\bar{x}$ to some point $x^{*} \in P$, and we suggest some possibilities to choose $x^{*}$. Essential concepts from convex analysis are gathered in an Appendix.

\section{Reverse polar and gauge}

In this section we consider a nonempty set $Q \subset \mathbb{R}^{n}$ (standing for $P-\bar{x}$ ), which we want to separate from the origin. As observed in the introduction, this amounts to finding a direction $d$ such that $\sigma_{Q}(d)<0$. Separating directions thus form an open set, which is delicate to manage numerically, when one wants to choose a distinguished element in it. The following definition, given in [1], ingeniously turns the difficulty around, taking advantage of positive homogeneity: the set

$$
Q^{-}:=\left\{d \in \mathbb{R}^{n}: \sigma_{Q}(d) \leqslant-1\right\}
$$

will be called the reverse polar of $Q$.

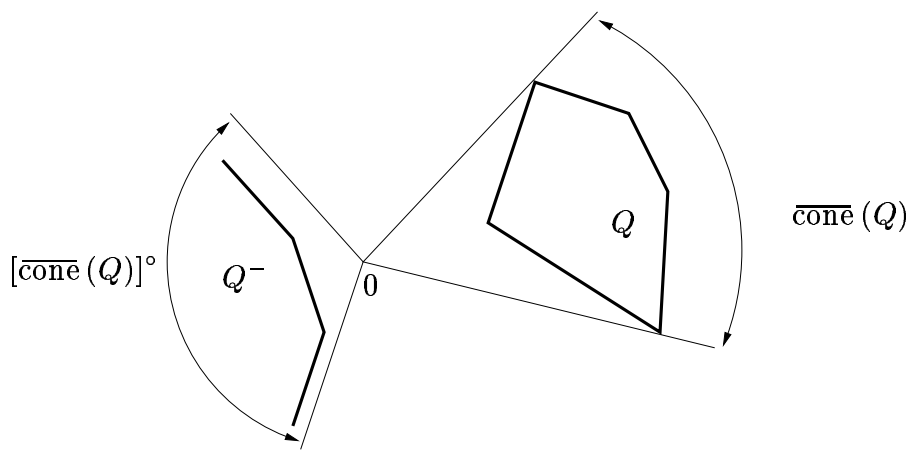

Figure 1: Reverse polar

Theorem 2.1 Assume $0 \notin \overline{\operatorname{conv}}(Q)$.

(i) The reverse polar $Q^{-}$is nonempty closed and convex.

(ii) Let lin $(Q)$ be the linear space generated by $Q$. Then $Q^{-}$has the form $R+(\operatorname{lin}(Q))^{\perp}$, where $R \subset \operatorname{lin}(Q)$.

(iii) A direction $d$ separates 0 from $Q$ if and only if $t d \in Q^{-}$for some $t>0$.

Proof. (i) Closedness and convexity hold because the function $\sigma_{Q}$ is itself closed and convex; see the Appendix for notation. Nonemptiness is just standard separation between $\overline{\operatorname{conv}}(Q)$ and $\{0\}$.

(ii) The property $\ell \perp \operatorname{lin}(Q)$ is equivalent to

$$
\ell^{\top} x=0 \text {, i.e. }(d+\ell)^{\top} x=d^{\top} x, \quad \text { for all } x \in Q .
$$

Thus $\sigma_{Q}(d)=\sigma_{Q}(d+\ell)$ and therefore $d \in Q^{-} \Leftrightarrow d+\ell \in Q^{-}$.

(iii) Finally, $d$ separates 0 from $Q$ means that $\sigma_{Q}(d) \leqslant-1 / t$, for some $t>0$; this is equivalent to $\sigma_{Q}(t d) \leqslant-1$, i.e. $t d \in Q^{-}$.

It is observed in [2] - and will be confirmed below - that, to compute good separating directions, it is advisable to maximize some linear function over the reverse polar set. This motivates the following definition and notation:

Definition 2.2 The support function of $Q^{-}$

$$
\mathbb{R}^{n} \ni q \mapsto \gamma_{Q}(q):=\sup _{\sigma_{Q}(d) \leqslant-1} q^{\top} d
$$

will be called the reverse gauge of $Q: \gamma_{Q}=\sigma_{Q^{-}}$.

The (possibly empty) optimal set is the face of $Q^{-}$exposed by $q$ :

$$
\Gamma_{Q}(q):=\mathrm{F}_{Q^{-}}(q)=\underset{d \in Q^{-}}{\operatorname{Argmax}} q^{\top} d .
$$


The following result goes along the lines of [3, Thm. 12].

Theorem 2.3 Assume $0 \notin \overline{\mathrm{conv}}(Q)$.

(i) The reverse gauge is a closed sublinear function.

(ii) Denoting by $\overline{\text { cone }}(Q)$ the closed conical hull of $Q$, there holds

$$
\gamma_{Q}(q) \begin{cases}\leqslant 0 & \text { if } q \in \overline{\operatorname{cone}}(Q) \\ =+\infty & \text { otherwise }\end{cases}
$$

(iii) If $q \in \overline{\operatorname{conv}}(Q)$ then $\gamma_{Q}(q) \leqslant-1$.

Proof. (i) Being a support function, $\gamma_{Q}$ is closed sublinear.

(ii) Use the notation $K:=\overline{\mathrm{cone}}(Q)$ and observe that $Q^{-} \subset K^{\circ}$ : if $q \in K$, then the objective value in (2) is nonpositive, as well as its supremum.

On the other hand, let $q \notin K$ : there exists $d_{0} \in K^{\circ}$ such that $q^{\top} d_{0}>0$ (separation between $q$ and $K$, see (14) in the Appendix). Note: $\sigma_{Q}\left(d_{0}\right) \leqslant \sigma_{K}\left(d_{0}\right) \leqslant 0$ (see (19) in the Appendix). Take $\bar{d} \in Q^{-} \subset K^{\circ}, \varepsilon>0$ and form the vector

$$
d(\varepsilon):=d_{0}+\varepsilon \bar{d} \subset K^{\circ} .
$$

For $\varepsilon$ small enough, $q^{\top} d(\varepsilon)>0$. By subadditivity of $\sigma_{Q}$, we also have

$$
\sigma_{Q}(d(\varepsilon)) \leqslant \sigma_{Q}\left(d_{0}\right)+\varepsilon \sigma_{Q}(\bar{d}) \leqslant-\varepsilon .
$$

Then, for $t \rightarrow+\infty$, we have

$$
\sigma_{Q}(t d(\varepsilon)) \rightarrow-\infty \quad \text { and } \quad q^{\top}(t d(\varepsilon)) \rightarrow+\infty .
$$

(iii) If $q \in \overline{\mathrm{conv}}(Q)$ then $q^{\top} d \leqslant \sigma_{Q}(d)$ for all $d$ (separation theorem, see (14) in the Appendix). Thus $q^{\top} d \leqslant-1$ for any feasible $d$ and this inequality is still valid for the supremum.

We finish this section with a comment on our nonemptiness assumption. The case $Q=\emptyset$ is somewhat trivial but also pathological: we may set $\sigma_{Q} \equiv-\infty$ (see Note 2, p.15) and it is natural from (1) to set $Q^{-}=\mathbb{R}^{n}$; then $\gamma_{Q}(0)=0$ and $\gamma_{Q}(d)=+\infty$ for all $d \neq 0$.

\section{Geometric characterizations}

This section is devoted to describing $\gamma_{Q}$ and $\Gamma_{Q}$ in terms of $Q$ itself, without any reference to $\sigma_{Q}$. This can be done via the following problem:

$$
\left\{\begin{array}{l}
\sup t \\
t \geqslant 0 \\
q \in t \overline{\operatorname{conv}}(Q)
\end{array}\right.
$$

and Theorem 3.2 below will justify our terminology "reverse gauge" (see the Appendix for the definition of a gauge).

In the remainder of this section, we will assume $q \in \overline{\mathrm{conv}}(Q)$, which is the case of interest for the applications we have in mind. Observe that, by positive homogeneity, this amounts to taking $q$ anywhere in the cone generated by $Q$; up to the closure operation, this essentially amounts to assuming $\gamma_{Q}(q)<+\infty$ (see Theorem 2.3 (ii)).

Lemma 3.1 Assume $0 \notin \overline{\mathrm{conv}}(Q)$ and let $q \in \overline{\mathrm{conv}}(Q)$. Then the optimal $t$ in $(3)$ is not smaller than 1 and the optimal value is attained (the sup is actually a max).

Proof. Call $t^{*}$ the optimal value of (3). The property $t^{*} \geqslant 1$ is obvious by our choice of $q$.

Let $t_{k}$ be a maximizing sequence: $q / t_{k} \in \overline{\mathrm{conv}}(Q)$ and $1 / t_{k} \rightarrow 1 / t^{*}$ (a finite number, possibly 0 ). Then $q / t_{k} \rightarrow q / t^{*}$, which has to lie in the closed set $\overline{\operatorname{conv}}(Q)$.

Theorem 3.2 Assume $0 \notin \overline{\mathrm{conv}}(Q)$ and let $q \in \overline{\mathrm{conv}}(Q)$. Then the optimal value in $(3)$ is $-\gamma_{Q}(q)$. 
Proof. Apply Lagrangian duality to (2) (a convex problem which satisfies Slater's condition):

$$
\gamma_{Q}(q)=\inf _{t \geqslant 0} \sup _{d \in \mathbb{R}^{n}}\left[q^{\top} d-t\left(\sigma_{Q}(d)+1\right)\right]=\inf _{t \geqslant 0}[-t+\theta(t)]
$$

where $\theta(t):=\sup _{d}\left[q^{\top} d-t \sigma_{Q}(d)\right]$. Observe that $\theta(t) \geqslant 0$ (just take $d=0$ ). Because $\gamma_{Q}(q) \leqslant-1$ (Theorem 2.3), we may impose the constraint $t \geqslant 1$. Then

$$
\theta(t)= \begin{cases}0 & \text { if } q \in t Q \\ +\infty & \text { otherwise }\end{cases}
$$

To see this, either invoke [10, $\S$ E.1] (more specifically Example 1.1.5 and Theorem 1.3.5) or argue as follows: by positive homogeneity $\theta(t)=+\infty$ if there is $d$ such that $q^{\top} d-t \sigma_{Q}(d)>0$, i.e. $q / t \notin Q$ (separation between $Q$ and $q / t,(14)$ in the Appendix). On the other hand $q / t \in Q$ means $\left(q^{\top} / t\right) d-\sigma_{Q}(d) \leqslant 0$ for all $d$ and then $\theta(t)=0$.

It follows that $\gamma_{Q}(t)=\inf \{-t: t \geqslant 0, q \in t Q\}$.

This result, together with Lemma 3.1, reveals important objects attached to $Q$ and $q$ :

Definition 3.3 Denoting by $t_{Q}^{*}(q)$ the optimal value of (3),

$$
q^{*}:=\frac{q}{-\gamma_{Q}(q)}=\frac{q}{t_{Q}^{*}(q)}
$$

is the visible point from the origin in the direction $q$.

Geometrically, $q^{*}$ is the closest point to the origin on the line-segment $[0, q]$, still lying in $\overline{\operatorname{conv}}(Q)$; see $[3$, Corr. 14]. In fact, (3) can also be written (setting $s:=1 / t$ ):

$$
\inf s, \quad s \geqslant 0, \quad s q \in \overline{\operatorname{conv}}(Q) .
$$

Clearly enough, $q \mapsto t_{Q}^{*}(q)$ is positively homogeneous: the length of $q$ has no real influence on (2) or (3) or $q^{*}$. The points $q \in \overline{\operatorname{conv}}(Q)$ that are visible from the origin are those such that $t_{Q}^{*}(q)=-\gamma_{Q}(q)=1$.

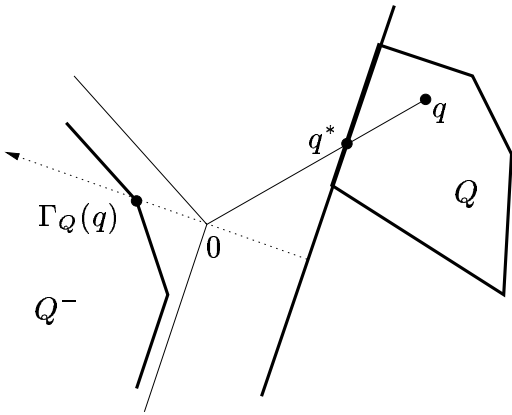

Figure 2: Visible point and exposed face

Theorem 3.4 Assume $0 \notin \overline{\mathrm{conv}}(Q)$, let $q \in \overline{\mathrm{conv}}(Q)$ and use the notation of Definition 3.3. Then $\Gamma_{Q}(q)$ is the set of $d$ in the normal cone to $\overline{\operatorname{conv}}(Q)$ at $q^{*}$ that satisfy $q^{\top} d=\gamma_{Q}(q)$. In other words, $d \in \Gamma_{Q}(q)$ is equivalent to

$$
\begin{aligned}
& d^{\top}\left(x-q^{*}\right) \leqslant 0 \text { for all } x \in Q \\
& \text { and } d^{\top} q=\gamma_{Q}(q) .
\end{aligned}
$$

Proof. Let $d$ satisfy (4); maximizing over $x \in Q$ shows that $\sigma_{Q}(d) \leqslant d^{\top} q^{*}$. If, in addition, $d$ satisfies (5), $\sigma_{Q}(d) \leqslant-1: d$ is feasible in (2); use again (5) to conclude that $d$ is also optimal.

Conversely, let $d \in \Gamma_{Q}(q)$; then $\sigma_{Q}(d) \leqslant-1$ and $q^{\top} d=\gamma_{Q}(q)$, hence

$$
\sigma_{Q}(d) \leqslant-1=d^{\top} q^{*} .
$$


However, $q^{*} \in \overline{\mathrm{conv}}(Q)$ (Lemma 3.1 and Theorem 3.2). By definition of a support function, $\sigma_{Q}\left(q^{*}\right) \geqslant d^{\top} q^{*}$ : by (6), we conclude (see (15) in the Appendix)

$$
q^{*} \in \mathrm{F}_{\overline{\operatorname{conv}}(Q)}(d), \quad \text { i.e. } \quad d \in \mathrm{N}_{\overline{\operatorname{conv}}(Q)}\left(q^{*}\right),
$$

where the last equivalence is (17) in the Appendix.

The meaning of this result is that a direction $d \in \Gamma_{Q}(q)$ exposes $q^{*}$ in $\overline{\operatorname{conv}}(Q)$. Said differently, $d \in \Gamma_{Q}(q)$ defines a hyperplane

$$
\left\{x \in \mathbb{R}^{n}: d^{\top} x=\sigma_{Q}(d)\right\}
$$

that supports $\overline{\operatorname{conv}}(Q)$ at $q^{*}$. The important property to characterize $\Gamma_{Q}(q)$ is $(4)$, while (5) just serves to specify the length of $d$.

The constructions given in this section are intrinsic: they do not rely on any representation of $Q$. It can be mentioned that the dilation effect revealed in (3) may result in rather intricate calculations when $Q$ is represented by nonlinear constraints - see [7]. For affine constraints, the calculations considerably simplify see $\S 6$ below, more specifically Remark 6.5.

\section{Normalizations}

Computing the reverse gauge can also be done via an optimization problem with a single linear constraint, namely:

$$
\inf \sigma_{Q}(d), \quad q^{\top} d \geqslant-1 ;
$$

we will denote by $\kappa^{*}$ and $D^{*}$ its optimal value and optimal set. Observe that (7) is just (2) with the objective function and constraint interchanged.

It is not difficult to see that the constraint in (2) or (7) can be turned to an equality:

Lemma 4.1 Assume $0 \notin \overline{\operatorname{conv}}(Q)$ and let $q \in \overline{\operatorname{conv}}(Q)$. Then:

(i) $\gamma_{Q}(q)$ and $\Gamma_{Q}(q)$ are the optimal value and optimal set of

$$
\max q^{\top} d, \quad \sigma_{Q}(d)=-1 ;
$$

(ii) $\kappa^{*}<0$ and $D^{*}$ are the optimal value and optimal set of

$$
\min \sigma_{Q}(d), \quad q^{\top} d=-1 .
$$

Proof. Let $d$ be feasible in (2): $\sigma_{Q}(d)=: \alpha \leqslant-1$; by definition of a support function, $q^{\top} d \leqslant \sigma_{Q}(d)<0$. By positive homogeneity, $\sigma_{Q}\left(\frac{d}{-\alpha}\right)=-1$ and $q^{\top} \frac{d}{-\alpha} \geqslant q^{\top} d$, with strict inequality if $-\alpha>1$. This proves (i). The proof of (ii) goes the same way.

Up to positive homogeneity, (2) and (7) are actually equivalent:

Theorem 4.2 Assume $0 \notin \overline{\mathrm{conv}}(Q)$ and let $q \in \overline{\mathrm{conv}}(Q)$. Then

$$
\gamma_{Q}(q)=\frac{1}{\kappa^{*}} \quad \text { and } \quad \Gamma_{Q}(q)=\frac{D^{*}}{-\kappa^{*}} .
$$

Proof. We use the shortened notation $\gamma:=\gamma_{Q}(q)$. Recall that $\gamma \leqslant-1$ (Theorem 2.3 (iii)).

Let $\varepsilon>0$. In view of Lemma 4.1(i), we can take $d_{\varepsilon}$ such that $\sigma_{Q}\left(d_{\varepsilon}\right)=-1$ and $q^{\top} d_{\varepsilon} \geqslant \gamma-\varepsilon$. Then set $d:=\frac{d_{\varepsilon}}{-\gamma+\varepsilon}$, so that $q^{\top} d \geqslant-1: d$ is feasible in (7), hence

$$
\kappa^{*} \leqslant \sigma_{Q}(d)=\frac{1}{-\gamma+\varepsilon} \sigma_{Q}\left(d_{\varepsilon}\right)=\frac{1}{\gamma-\varepsilon} .
$$

Since $\varepsilon>0$ is arbitrary, $\kappa^{*} \leqslant 1 / \gamma$, i.e. $\kappa^{*} \gamma \geqslant 1$, i.e. $\gamma \leqslant 1 / \kappa^{*}$.

On the other hand, Lemma 4.1(ii) allows us to force $\varepsilon \in] 0,-\kappa^{*}\left[\right.$ and to take $d_{\varepsilon}^{\prime}$ such that $q^{\top} d_{\varepsilon}^{\prime}=-1$ and $\sigma_{Q}\left(d_{\varepsilon}^{\prime}\right) \leqslant \kappa^{*}+\varepsilon<0$. Then set $d:=\frac{d_{\varepsilon}^{\prime}}{-\kappa^{*}-\varepsilon}$, so that $\sigma_{Q}(d) \leqslant-1: d$ is feasible in (2), hence

$$
\gamma \geqslant q^{\top} d=q^{\top} \frac{d_{\varepsilon}^{\prime}}{-\kappa^{*}-\varepsilon}=\frac{1}{\kappa^{*}+\varepsilon} .
$$


Since $\varepsilon>0$ is arbitrarily small, $\gamma \geqslant 1 / \kappa^{*}$. Altogether, $\gamma=1 / \kappa^{*}$.

Now $d \in \Gamma_{Q}(q)$ means

$$
q^{\top} d=\gamma \quad \text { and } \quad \sigma_{Q}(d)=-1,
$$

while $d \in D^{*}$ means

$$
q^{\top} d=-1 \quad \text { and } \quad \sigma_{Q}(d)=\frac{1}{\gamma} .
$$

The relation between $\Gamma_{Q}(q)$ and $D^{*}$ follows by positive homogeneity.

With reference to our introduction, the constraint in (7) can be viewed as a normalization: to construct a tightest cut $d$ amounts to minimizing $\sigma_{Q}$ and requires a bound on $d$. The above result establishes that bounding $q^{\top} d$ (from below) does the job: it imposes the simplest possible constraint to give a meaning to the minimization of $\sigma_{Q}$. It does not necessarily produce a separating direction, though: consider in $\mathbb{R}^{2}$ the circle

$$
Q:=\left\{x=(\xi, \eta):(\xi-1)^{2}+(\eta-1)^{2} \leqslant 1\right\}
$$

and take $q=(1,0)$. Then $\gamma_{Q}(q)=-1$ (Theorem 3.2). However, no $d \in\left(0, \mathbb{R}_{-}\right)=\mathrm{N}_{Q}(q)$ satisfies $d^{\top} q=-1$ : in fact $\Gamma_{Q}(q)=\emptyset$.

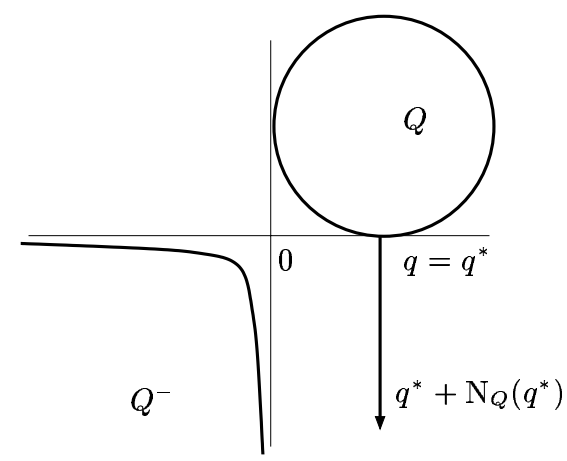

Figure 3: No separator exposes $q^{*}$

Remark 4.3 The proof of Theorem 3.2 shows that (3) is the Lagrangian dual of (2). A similar interpretation applies to (7) through the following formulation of (3):

$$
\inf _{x \in Q, t \geqslant 0} t, \quad t q=x .
$$

Dualizing the constraint $t q-x=0$ with multiplier $d$, we obtain the Lagrangian dual

$$
\sup _{d \in \mathbb{R}^{n}} \inf _{x \in Q, t \geqslant 0} t+d^{\top}(t q-x) .
$$

The internal inf-problem can be solved separately in $x$ and $t$. Remembering the definion of $\sigma_{Q}$, we obtain

$$
\sup _{d \in \mathbb{R}^{n}}-\sigma_{Q}(d), \quad q^{\top} d+1 \geqslant 0,
$$

which is nothing other than (7).

Note also the optimality condition of

$$
\inf _{x \in Q}-d^{\top} x,
$$

which is $d \in \mathrm{N}_{Q}(x)$ and "explains" Theorem 3.4.

\section{The case of a disjunction}

Let $Q_{0}$ and $Q_{1}$ be two nonempty subsets of $\mathbb{R}^{n}$. In this section, we take $Q=Q_{0} \cup Q_{1}$ and we apply the above results to compute the reverse elements of $Q$ in terms of the data $Q_{0}$ and $Q_{1}$. 
Lemma 5.1 Denote by $\sigma_{i}$ and $\gamma_{i}$ the support function and reverse gauge of $Q_{i}, i=0,1$. There holds

$$
\sigma_{Q}=\max \left\{\sigma_{0}, \sigma_{1}\right\}, \quad Q^{-}=Q_{0}^{-} \cap Q_{1}^{-}, \quad \gamma_{Q}=\overline{\operatorname{conv}}\left(\min \left\{\gamma_{0}, \gamma_{1}\right\}\right) .
$$

Proof. See the Appendix for $\sigma_{Q}$. The formula for $Q^{-}$follows easily. See again the Appendix for $\gamma_{Q}=\sigma_{Q^{-}}$.

In the following result, the notation $\bar{S}$ is used for the closure of a set $S$.

Theorem 5.2 Assume $0 \notin \overline{\operatorname{conv}}(Q)$ and let $q \in \overline{\operatorname{conv}}(Q)$. Then $-\gamma_{Q}(q)$ is the optimal value of

$$
\left\{\begin{array}{l}
\sup t_{0}+t_{1} \\
t_{0} \geqslant 0, t_{1} \geqslant 0 \\
q \in \overline{t_{0} \operatorname{conv}\left(Q_{0}\right)+t_{1} \operatorname{conv}\left(Q_{1}\right)}
\end{array}\right.
$$

Proof. Apply Theorem 3.2: $-\gamma_{Q}(q)$ is the optimal value of (3). Now $t \overline{\operatorname{conv}}(Q)$ is clearly $\overline{c o n v}(t Q)$, which is the closure of $S_{t}:=\operatorname{conv}(t Q)$. Using associativity of convex combinations, we can write

$$
S_{t}=\left\{\alpha t q_{0}+(1-\alpha) t q_{1}: \alpha \in[0,1], q_{0} \in \operatorname{conv}\left(Q_{0}\right), q_{1} \in \operatorname{conv}\left(Q_{1}\right)\right\} .
$$

Introduce the notation $t_{0}:=\alpha t, t_{1}:=(1-\alpha) t$. When $\alpha$ describes the segment $[0,1], t_{0}$ and $t_{1}$ describe the corresponding segment dilated by $t \geqslant 0$, so that

$$
S_{t}=\left\{t_{0} \operatorname{conv}\left(Q_{0}\right)+t_{1} \operatorname{conv}\left(Q_{1}\right): t_{0} \geqslant 0, t_{1} \geqslant 0, t_{0}+t_{1}=t\right\} .
$$

Introducing the visible point $q^{*}$ of Definition 3.3 allows the characterization of $\Gamma_{Q}(q)$ in terms of normal cones (see (16) in the Appendix):

Theorem 5.3 Assume $0 \notin \overline{\operatorname{conv}}(Q)$ and let $q \in \overline{\operatorname{conv}}(Q)$. Then $\Gamma_{Q}(q)$ is the set of $d$ satisfying

$$
\begin{aligned}
& d^{\top}\left(x-q^{*}\right) \leqslant 0 \text { for all } x \in Q_{0}, \\
& d^{\top}\left(x-q^{*}\right) \leqslant 0 \text { for all } x \in Q_{1}, \\
& \text { and } q^{\top} d=\gamma_{Q}(q) .
\end{aligned}
$$

Proof. Use Theorem 3.4 and observe that, if $d$ satisfies $d^{\top}\left(x-q^{*}\right) \leqslant 0$ for all $x \in Q_{i}, i=0,1$, then the same inequality holds

- for all $x \in \operatorname{conv}\left(Q_{0} \cup Q_{1}\right)$ by convex combinations,

- and for all $x$ in the closure of the latter set, by continuity.

Altogether, (4) is satisfied. The converse is obvious.

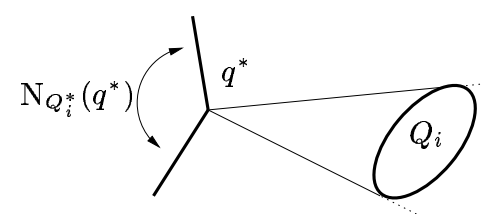

Figure 4: Apexed convex hull and corresponding normal cone

Remark 5.4 This results suggests that the normal cone $\mathrm{N}_{Q}\left(q^{*}\right)$ (where $Q$ is a union) is the intersection of two appropriate normal cones, as illustrated by Fig. 4: for $i=1,2$, we have

$$
\begin{aligned}
Q_{i}^{*}:=\operatorname{conv}\left(Q_{i} \cup\left\{q^{*}\right\}\right) & =\left\{(1-\alpha) q^{*}+\alpha x: x \in Q_{i}, \alpha \in[0,1]\right\} \\
& =q^{*}+\left\{\alpha\left(Q_{i}-q^{*}\right): \alpha \in[0,1]\right\} .
\end{aligned}
$$

It follows that the directions $d$ satisfying

$$
d^{\top}\left(x-q^{*}\right) \leqslant 0 \text { for all } x \in Q_{i},
$$


or equivalently

$$
d^{\top} \alpha\left(x-q^{*}\right) \leqslant 0 \text { for all } x \in Q_{i} \text { and } \alpha \in[0,1]
$$

just make up the normal cone $\mathrm{N}_{Q_{i}^{*}}\left(q^{*}\right)$ to $Q_{i}^{*}$ at $q^{*}$. As a result, $d \in \Gamma_{Q}(q)$ means

$$
\begin{aligned}
& d \in \mathrm{N}_{Q_{0}^{*}}\left(q^{*}\right) \cap \mathrm{N}_{Q_{1}^{*}}\left(q^{*}\right) \\
& \text { and } q^{\top} d=\gamma_{Q}(d) .
\end{aligned}
$$

The whole issue when dealing with a disjunction is thus to characterize a normal cone of the type $\mathrm{N}_{\text {conv }(S \cup\{0\})}(0)$, for some set $S$ (here $S=Q_{i}-q^{*}$ ). Such a cone can also be viewed as the polar of cone $(S)$.

One can also compute the reverse elements of a disjunction using a normalization, as in $\S 4$ :

Theorem 5.5 Denote by $\kappa^{*}$ and $D^{*}$ the optimal value and optimal set (possibly empty) of

$$
\inf \kappa, \quad \kappa \geqslant \sigma_{0}(d), \quad \kappa \geqslant \sigma_{1}(d), \quad q^{\top} d=-1 \text {. }
$$

Then $\gamma_{Q}(q)=\frac{1}{\kappa^{*}}$ and $\Gamma_{Q}(q)=\frac{D^{*}}{-\kappa^{*}}$.

Proof. Use Lemma 5.1 in (7): the result is a straigtforward application of Theorem 4.2.

\section{Polyhedra}

A polyhedron can be described in two different ways: as a convex hull (Theorem 6.1 below), or as an intersection of half-spaces, as in $\S 6.1$. The case of a disjunction $(\S 6.2)$ is somewhat intermediate.

Theorem 6.1 Let $Q$ be a nonempty closed convex polyhedron not containing the origin. Then $Q^{-}$is also a nonempty closed convex polyhedron; in particular, if $Q=\operatorname{conv}\left(x^{1}, \ldots, x^{k}\right)+\operatorname{cone}\left(r^{1}, \ldots, r^{\ell}\right)$, then

$$
d \in Q^{-} \Longleftrightarrow \begin{cases}d^{\top} x^{i} \leqslant-1 & \text { for } i=1, \ldots, k, \\ d^{\top} r^{j} \leqslant 0 & \text { for } j=1, \ldots, \ell .\end{cases}
$$

Furthermore, $\gamma_{Q}$ is a piecewise linear function in the domain where it is finite; $\Gamma_{Q}(q)$ is a closed convex polyhedron, nonempty if $q \in \overline{\mathrm{cone}}(Q)=$ cone $(Q)$.

Proof. Everything is rather clear; in particular, (2) is a linear program, which has a nonempty optimal set if it has a finite optimal value.

Now comes a result motivating this theory.

Theorem 6.2 The face $\mathrm{F}_{Q}(d)$ exposed by $d \in Q^{-}$is a facet of $Q$ if and only if

$$
d^{*}:=\frac{d}{-\sigma_{Q}(d)}
$$

is an extreme point of $Q^{-} \cap \operatorname{lin}(Q)$.

Proof. The points $x \in Q$ that lie in $\mathrm{F}_{Q}(d)$ are those such that $d^{\top} x=\sigma_{Q}(d)$, i.e. $\left(d^{*}\right)^{\top} x=-1$.

Let $p$ denote the dimension of $\operatorname{lin}(Q)^{\perp}$. Suppose $\mathrm{F}_{Q}(d)$ is a facet of $Q$ and let $x^{1}, \ldots, x^{n-p}$ be $n-p$ affinely independent points in $\mathrm{F}_{Q}(d)$. Then $d^{*}$ satisfies a system of $n-p$ independent equations $\left(d^{*}\right)^{\top} x^{i}=-1$ which uniquely determines it up to the linear space $\operatorname{lin}(Q)^{\perp}$. Conversely, if $d^{*}$ is an extreme point of $Q^{-} \cap$ lin $(Q)$, there exist $n-p$ affinely independent points $x^{1}, \ldots, x^{n-p}$ in $Q$ that satisfy $\left(d^{*}\right)^{\top} x^{i}=-1$. These points lie in $\mathrm{F}_{Q}(d)$, therefore $\mathrm{F}_{Q}(d)$ is a facet of $Q$. 


\subsection{Explicitly described polyhedra}

Assume that $Q$ is described by a set of constraints:

$$
Q=\left\{x \in \mathbb{R}^{n}: A x \leqslant b \in \mathbb{R}^{m}\right\} .
$$

A simple way of computing $\gamma_{Q}$ is then as follows:

Theorem 6.3 Assume $Q$ of (8) does not contain the origin and let $q \in Q$; denote by $A_{j}$ the $j^{\text {th }}$ row of $A$. Then

$$
-\gamma_{Q}(q)=\max _{b_{j}<0} \frac{A_{j} q}{b_{j}} .
$$

Calling $J(q)$ the set of $j$ realizing the above $\max , \Gamma_{Q}(q)$ is the set of directions in cone $\left(A_{j}\right)_{j \in J(q)}$, normalized by the constraint $d^{\top} q=\gamma_{Q}(q)$.

Proof. Just apply Theorems 3.2 and 3.4: (3) takes the form

$$
\max t, \quad t \geqslant 0, \quad A q \leqslant t b .
$$

Then use the known expression for the normal cone to a polyhedron.

\subsection{Union of polyhedra}

Now take the situation of $\S 5: Q:=\overline{\mathrm{conv}}\left(Q_{0} \cup Q_{1}\right)$, where

$$
Q_{i}:=\left\{x \in \mathbb{R}^{n}: A_{i} x \leqslant b_{i} \in \mathbb{R}^{m_{i}}\right\} \quad \text { for } i=0,1
$$

are nonempty closed convex polyhedra. Of course, all the constructions of the present section can be obtained via appropriate linear programs. However, we will keep close to our convex-analysis point of view.

We start by a characterization of the closed convex hull $Q$ described above.

Lemma 6.4 With the notation (9), $x \in Q$ if and only if there are $y_{0}, y_{1} \in \mathbb{R}^{n}$ and $\alpha_{0}, \alpha_{1} \in \mathbb{R}$ such that

$$
x=y_{0}+y_{1}, \quad \alpha_{0}+\alpha_{1}=1, \quad A_{i} y_{i}-\alpha_{i} b_{i} \leqslant 0 \text { and } \alpha_{i} \geqslant 0 \text { for } i=0,1 .
$$

Proof. Consider the set

$$
S:=\left\{\alpha_{0} x_{0}+\alpha_{1} x_{1}: \alpha_{0}+\alpha_{1}=1, A_{i} x_{i} \leqslant b_{i} \text { and } \alpha_{i}>0 \text { for } i=0,1\right\} .
$$

Clearly enough, $S \subset \operatorname{conv}\left(Q_{0} \cup Q_{1}\right)$ and any point in conv $\left(Q_{0} \cup Q_{1}\right)$ can be approximated to any accuracy by a point in $S$. It follows that $Q$ is the closure of $S$.

Now set $y_{i}:=\alpha_{i} x_{i}$ for $i=0,1$, so that we have

$$
S=\left\{y_{0}+y_{1}: \alpha_{0}+\alpha_{1}=1, A_{i} y_{i} \leqslant \alpha_{i} b_{i} \text { and } \alpha_{i}>0 \text { for } i=0,1\right\},
$$

whose closure is clearly the set described by (10).

This result will be used to characterize $t Q$ in $\S 3$ by appropriate constraints.

Remark 6.5 The above proof makes more precise our comments at the end of §3. In fact, suppose $Q=\{x$ : $c(x) \leqslant 0\}$ (with $c$ convex but not necessarily affine). Then the feasible set in (3) is given by $c(q / t) \leqslant 0$, a nasty nonconvex constraint (with respect to the variable $(q, t)$ ). Here comes a trick: with $t>0$ we can also write $t c(q / t) \leqslant 0$ and this reveals the so-called perspective function of $c$ (see [10]):

$$
\left.\mathbb{R}^{n} \times\right] 0,+\infty\left[\ni(q, t) \mapsto \tilde{c}(q, t):=t c\left(\frac{q}{t}\right),\right.
$$

which happens to be convex (and even sublinear, see the Appendix). When $c$ is affine, $\tilde{c}$ is linear, which results in the simple formula (10). Otherwise, $\tilde{c}$ is rather involved, which explains the technicalities developed in [7]. 
Theorem 6.6 With the notation (9), assume $0 \notin Q$ and let $q \in Q$. Then $-\gamma_{Q}(q)$ is the optimal value of

$$
\begin{cases}\max t_{0}+t_{1} & \\ q_{0}+q_{1}=q & \\ A_{i} q_{i}-t_{i} b_{i} \leqslant 0 & \text { for } i=0,1 \\ t_{i} \geqslant 0 & \text { for } i=0,1\end{cases}
$$

Proof. Just dilate the set (10) by $t \geqslant 0$, replacing $\alpha_{i}$ by $t_{i}:=t \alpha_{i}$.

There remains to characterize $\Gamma_{Q}(q)$, i.e. the normal cone to $Q$ at $q^{*}$. The following result, inspired by Remark 5.4, will allow the application of Theorem 5.3.

Proposition 6.7 With the notation (9) and $q^{*}$ of Definition 3.3, the cone

$$
N_{0}:=\left\{d \in \mathbb{R}^{n}: d^{\top}\left(x-q^{*}\right) \leqslant 0 \text { for all } x \in Q_{0}\right\}
$$

is the set of $d=A_{0}^{\top} u_{0}$, where $u_{0} \geqslant 0 \in \mathbb{R}^{m_{0}}$ satisfies $\left(A_{0}^{\top} q^{*}-b_{0}\right)^{\top} u_{0} \geqslant 0$.

Proof. Using an artificial multiplication by $\alpha>0$, we can write that $d \in N_{0}$ if and only if

$$
\alpha d^{\top}\left(x-q^{*}\right) \leqslant 0 \text { for all } x \text { such that } A_{0} x \leqslant b_{0} \text { and all } \alpha \geqslant 0
$$

which, setting $y:=\alpha x-\alpha q^{*}$, can also be written

$$
d^{\top} y \leqslant 0 \text { for all }\left(\begin{array}{c}
y \\
\alpha
\end{array}\right) \in K_{0}
$$

here $K_{0} \subset \mathbb{R}^{n+1}$ is the cone defined by $\left(\begin{array}{cc}A_{0} & A_{0} q^{*}-b_{0} \\ 0^{\top} & -1\end{array}\right)\left(\begin{array}{l}y \\ \alpha\end{array}\right) \leqslant 0$. This means that $\left(\begin{array}{c}d \\ 0\end{array}\right) \in K_{0}^{\circ}$.

Now $K_{0}^{\circ}$ is classically the set described by

$$
\left(\begin{array}{l}
d \\
r
\end{array}\right)=\left(\begin{array}{cc}
A_{0}^{\top} & 0 \\
\left(A_{0} q^{*}-b_{0}\right)^{\top} & -1
\end{array}\right)\left(\begin{array}{l}
u_{0} \\
v_{0}
\end{array}\right) \quad \text { with }\left(\begin{array}{l}
u_{0} \\
v_{0}
\end{array}\right) \geqslant 0 \in \mathbb{R}^{n+1}
$$

Fixing $r=0$ introduces the constraint

$$
\left(A_{0} q^{*}-b_{0}\right)^{\top} u_{0}-v_{0}=0 \text {, i.e. }\left(A_{0} q^{*}-b_{0}\right)^{\top} u_{0} \geqslant 0 \text {. }
$$

The characterization of $\Gamma_{Q}(q)$ is now easy:

Theorem 6.8 With the notation (9), assume $0 \notin Q$ and let $q \in Q$; introduce $\gamma_{Q}(q)$ of (11) and $q^{*}$ of Definition 3.3. Up to the normalization $d^{\top} q^{*}=\gamma_{Q}(q), \Gamma_{Q}(q)$ is the set of $d=A_{0}^{\top} u_{0}=A_{1}^{\top} u_{1}$, with $\left(u_{0}, u_{1}\right) \in$ $\mathbb{R}_{+}^{m_{0}} \times \mathbb{R}_{+}^{m_{1}}$ satisfying the set of constraints

$$
A_{0}^{\top} u_{0}=A_{1}^{\top} u_{1} \quad \text { and } \quad\left(A_{i} q^{*}-b_{i}\right)^{\top} u_{i} \geqslant 0, i=0,1
$$

Proof. Straightforward from Theorem 5.3 and Proposition 6.7.

Remark 6.9 It has been observed in Remark4.3 that $\Gamma_{Q}(q)$ could also be obtained by applying duality to (3), which is here (11). Indeed, multiply the objective function of (3) by some normalizing coefficient $\kappa>0$ and call $d$ the multiplier associated with $q_{0}+q_{1}=q$. This gives the dual

$$
\begin{cases}\min -q^{\top} d & \\ d=A_{i}^{\top} u_{i} & \text { for } i=0,1 \\ \kappa+b_{i}^{\top} u_{i} \leqslant 0 & \text { for } i=0,1 \\ u \geqslant 0 & \end{cases}
$$

Its solution depends multiplicatively on $\kappa$, which can be adjusted so as to reproduce $q^{\top} d=\gamma_{Q}(q)$. The "correct" value is a posteriori $\kappa=-\left(A_{0} q^{*}\right)^{\top} u_{0}=-\left(A_{1} q^{*}\right)^{\top} u_{1}$. 


\section{Conclusion}

This paper has studied the construction of cutting planes defined by $\left(d, \beta=\sigma_{Q}(d)\right) \in \mathbb{R}^{n} \times \mathbb{R}$, separating the origin from (the closed convex hull of) a set $Q$. With regard to our introduction, this is also the problem of separating a point $\bar{x}$ from (the closed convex hull of) a set $P$ : it suffices to set $Q:=P-\bar{x}$, and to use the obvious relation $\sigma_{Q}(d)=\sigma_{P}(d)-d^{\top} \bar{x}$.

The $d$-part of a cut is conveniently computed with the help of some $q \in \overline{\operatorname{conv}}(Q)$, or rather its corresponding visible point $q^{*}$. We solve (2) or (7) which, in the translated context, take the form

$$
\sup _{\sigma_{P}(d)-d^{\top} \bar{x} \leqslant-1} d^{\top}(\underline{x}-\bar{x}) \quad \text { or } \quad \inf _{d^{\top}(\underline{x}-\bar{x}) \geqslant-1} \sigma_{P}(d)-d^{\top} \bar{x}
$$

(here $\underline{x}-\bar{x}$ stands for $q$, with $\underline{x} \in P$ ).

We have not considered the problem of choosing $q$ in $Q$ - i.e. $\underline{x}$ in $P$; but some suggestions can be made. A guide for this choice might be the depth of a cut, defined as follows.

Definition 7.1 The depth of a cut separating $\bar{x}$ from a closed convex set $P$ is the Euclidean distance between $\bar{x}$ and the separating hyperplane. If the cut is defined by

$$
d^{\top} x=\sigma_{P-\bar{x}}(d), \quad \text { where } \sigma_{P-\bar{x}}(d)=\sigma_{P}(d)-d^{\top} \bar{x}<0,
$$

its depth is $\frac{-\sigma_{P-\bar{x}}(d)}{\|d\|}$.

In fact, P. Bonami [5] performed experiments in the context of lift-and-project cuts to solve $\max c^{\top} x$ over $S=\left\{x \in\{0,1\}^{n}: A x \leqslant b\right\}$. He compared two choices of $q=\underline{x}-\bar{x}$ by choosing $\underline{x} \in S$ in two different ways: in the first, $\underline{x}$ was chosen to be a good heuristic solution to the integer program and in the second $\underline{x}$ was a bad solution (obtained by minimizing $c^{\top} x$ instead of maximizing it). One would expect the direction $q$ to be more central in cone $(Q)$ in the second case. On 15 instances of Miplib $3.0^{1}$, the average depth of a cut generated by the first choice of $\underline{x}$ was 0.026 whereas it was 0.084 with the second choice. Adding cuts for all $0-1$ variables $x_{j}$ where $\bar{x}_{j}$ was fractional gave an average improvement in objective value of $6 \%$ (of the gap between the linear and integer program objective values) in the first case and $6.6 \%$ in the second case. When the cuts were added to $A x \leqslant b$ and this strengthened linear program was solved again to compute a new solution $\bar{x}$, this process being repeated iteratively for one minute of CPU, the difference was even more significant ( $38 \%$ versus $53 \%$ ).

This small experiment suggests that it seems preferable to choose deep cuts, and one may therefore wish to find the deepest one; this corresponds to projecting $\bar{x}$ onto $P$ :

Proposition 7.2 With the above notation, call $x^{*}$ the solution of

$$
\min _{x \in P}\|x-\bar{x}\| \text {. }
$$

Then $d^{*}:=\bar{x}-x^{*}$ defines the deepest cut between $\bar{x}$ and $P$.

Proof. Using positive homogeneity, a cut $(d, \beta)$ can be restricted to a normalized $d$. Thus, a deepest cut is a solution of

$$
\min _{\|d\|=1} \sigma_{P-\bar{x}}(d)
$$

where, because the optimal objective value is negative, the constraint $\|d\|=1$ can be replaced by $\|d\| \leqslant 1$. Thus, a deepest cut is a solution of

$$
\min _{\|d\| \leqslant 1} \sup _{x \in P} d^{\top}(x-\bar{x}) .
$$

This is the min-maximization of a bilinear function over a product of closed convex sets, one of which (the unit ball) is compact. Using a standard minimax theorem, for example [12, Corollary 37.3.2], we can equivalently solve

$$
\sup _{x \in P} \min _{\|d\| \leqslant 1} d^{\top}(x-\bar{x}) .
$$

For given $x \in P$ (and hence different from $\bar{x}$ ), the internal minimization gives

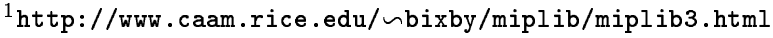

$$
d=d(x)=-\frac{x-\bar{x}}{\|x-\bar{x}\|}
$$


and our minimax problem boils down to

$$
\sup _{x \in P}-\|x-\bar{x}\|,
$$

whose unique solution is clearly the projection $x^{*}$ of $\bar{x}$ onto $P$.

Thus, to construct the deepest cut between a closed convex set $P$ and a point $\bar{x} \notin P$, one needs to project $\bar{x}$ onto $P$. When $P$ is a polyhedron, this amounts to solving a linear-quadratic problem.

When solving an integer program by a cutting plane approach, generating just one cut is usually not enough, even the deepest one. A better strategy is to generate many cuts at once; and as already mentioned, these should preferably be facets of $P$. An attractive approach is therefore to operate as follows:

(i) Compute the projection $x^{*}$ of $\bar{x}$ onto $P$. Set $d^{*}=\bar{x}-x^{*}$.

(ii) Construct cuts, say

$$
\left(d_{k}, \sigma_{P}\left(d_{k}\right)-\bar{x}^{\top} d_{k}\right), \quad k=1, \ldots, K
$$

corresponding to facets of $P$ passing through $x^{*}$.

(iii) Append to the above list $\left(d^{*}, \sigma_{P}\left(d^{*}\right)-\bar{x}^{\top} d^{*}\right)$ (this step is useless if (ii) has produced enough cuts, so that $d^{*}$ is a positive combination of the $d_{k}$ 's).

Assume for example the situation of $\S 6.2: P=\overline{\operatorname{conv}}\left(P_{0} \cup P_{1}\right)$ where

$$
P_{i}:=\left\{x \in \mathbb{R}^{n}: A_{i} x \leqslant b_{i} \in \mathbb{R}^{m_{i}}\right\} \text { for } i=0,1
$$

According to Lemma 6.4, (i) amounts to solving the problem with $2 n+2$ variables and $1+m_{0}+m_{1}$ constraints

$$
\left\{\begin{array}{l}
\min \frac{1}{2}\left\|y_{0}+y_{1}-\bar{x}\right\|^{2} \\
\alpha_{0}+\alpha_{1}=1 \\
A_{i} y_{i}-\alpha_{i} b_{i} \leqslant 0 \\
\alpha \geqslant 0,
\end{array} \quad \text { for } i=0,1\right.
$$

which produces an optimal solution $x^{*}=y_{0}^{*}+y_{1}^{*}$. Then Step (ii) should be done with the help of the active constraints; this, however, is not completely straightforward and is currently under study.

Let us also mention that Bonami [4] used the following idea for generating facets of $P$ that define the optimum of $\max _{x \in P} c^{\top} x$. First find $\bar{x}$ solving the relaxation $\max _{x \in R} c^{\top} x$ (we use the notation of the introduction). Then solve the linear program based on (11) to find a cut $\left(d_{1}, \sigma_{P}\left(d_{1}\right)-\bar{x}^{\top} d_{1}\right)$ separating $\bar{x}$ from $P$ (any $q \in Q$ will do in (11): for example $q=x^{*}-\bar{x}$ where, as above, $x^{*}$ is the projection of $\bar{x}$ onto $P$ ). Let $R^{1}$ be the relaxation obtained by adding this cut to $R$, and let $x^{1}$ maximize $c^{\top} x$ over $R^{1}$. The procedure is then repeated: the $k$ th iteration generates

- a cut $\left(d_{k}, \sigma_{P}\left(d_{k}\right)-\bar{x}^{\top} d_{k}\right)$ separating $x_{k-1}$ from $P$,

- an improved relaxation $R^{k}$ obtained by adding this cut to $R^{k-1}$,

- and a point $x^{k}$ maximizing $c^{\top} x$ over $R^{k-1}$.

The procedure terminates when $x^{k} \in P$. Bonami tried this procedure on the Miplib problems mentioned earlier, for the lift-and-project relaxation

$$
P_{L \& P}:=\bigcap_{j=1}^{n} \operatorname{conv}\left(\left(R \cap\left\{x_{j}=0\right\}\right) \cup\left(R \cap\left\{x_{j}=1\right\}\right)\right) .
$$

On average, maximizing $c^{\top} x$ over $P_{L \& P}$ closed $30 \%$ of the gap between $R$ and $\operatorname{conv}(S)$.

\section{Appendix: basic concepts from convex analysis}

We summarize here some material mainly extracted from [10, Chap. C].

Support functions An important object associated to a nonempty subset $S$ of $\mathbb{R}^{n}$ is its so-called support function

$$
\mathbb{R}^{n} \ni d \mapsto \sigma_{S}(d):=\sup _{x \in S} d^{\top} x
$$

In other words, $\sigma_{S}$ is the result of optimizing a linear function over $S$.

A support function enjoys the following important properties: 
(i) It is convex (being a supremum of linear functions ${ }^{2}$ ).

(ii) It is positively homogeneous: $\sigma_{S}(t d)=t \sigma_{S}(d)$ for all $t>0$, so that $d$ in (12) can be understood as a direction (knowing that $\sigma_{S}(0)=0$ ).

Functions that are convex and positively homogeneous are called sublinear; they are in particular subadditive, i.e.

$$
\sigma_{S}\left(d_{1}+d_{2}\right) \leqslant \sigma_{S}\left(d_{1}\right)+\sigma_{S}\left(d_{2}\right) .
$$

Note also that $\sigma_{S}$ is linear if and only if $S$ is a single point. Being convex, $\sigma_{S}$ would be continuous if it were finite everywere. However, we may have $\sigma_{S}(d)=+\infty$ (when $S$ is unbounded; for example $\sigma_{\mathbb{R}^{n}}(d$ ) is 0 if $d=0$ and $+\infty$ otherwise).

(iii) Nevertheless, a support function is lower-semicontinuous, or closed $^{3}$.

The set of functions that are sublinear and closed is important, as it directly generalizes the set of linear functions.

Correspondence between analysis and geometry Let $\overline{\operatorname{conv}}(S)$ denote the closed convex hull of $S$, equivalently defined as

- the topological closure of the convex hull of $S$ (the closure operation is superfluous if $S$ is already closed and bounded)

- or the intersection of all the half-spaces containing $S$.

Recall that a half-space associated to $(d, \beta) \in \mathbb{R}^{n} \times \mathbb{R}$ is defined as

$$
\left\{x \in \mathbb{R}^{n}: d^{\top} x \leqslant \beta\right\} .
$$

It is clear enough that $\sigma_{S}=\sigma_{\overline{\mathrm{conv}}(S)}$, and this is the starting remark for a fundamental result: the closed convex sets $S$ in $\mathbb{R}^{n}$ are in one-to-one correspondence with the functions $\sigma$ from $\mathbb{R}^{n}$ to $\mathbb{R} \cup\{+\infty\}$ that are closed and sublinear. The correspondence $S \mapsto \sigma$ is given by (12); its inverse is given by

$$
\sigma \mapsto S:=\left\{x \in \mathbb{R}^{n}: \sigma(d) \geqslant x^{\top} d \text { for all } d \in \mathbb{R}^{n}\right\}=\partial \sigma(0),
$$

where the last equality is obvious, if one remembers the definition of a subdifferential:

$$
\partial \sigma(\bar{d}):=\left\{x \in \mathbb{R}^{m}: \sigma(d) \geqslant \sigma(\bar{d})+x^{\top}(d-\bar{d}) \text { for all } d \in \mathbb{R}^{n}\right\} .
$$

From the definition (12) itself, $d^{\top} x \leqslant \sigma_{S}(d)$ for all $d \in \mathbb{R}^{n}$ whenever $x \in \overline{\mathrm{conv}}(S)$. The above result states that the converse is true:

$$
x \in \overline{\operatorname{conv}}(S) \quad \Longleftrightarrow \quad \forall d \in \mathbb{R}^{n}, d^{\top} x \leqslant \sigma_{S}(d),
$$

which is nothing other than the familiar separation theorem

$$
x \notin \overline{\operatorname{conv}}(S) \quad \Longleftrightarrow \quad \exists d \in \mathbb{R}^{n}: d^{\top} x>\sigma_{S}(d) .
$$

Isomorphic aspects Not only closed convex sets and closed sublinear functions, but also operations between them are in correspondence via (12) and (13), which is indeed an isomorphism. For example,

$$
\sigma_{S_{1}+S_{2}}=\sigma_{S_{1}}+\sigma_{S_{2}} .
$$

Two other important operations are as follows: given an index set $J$ (possibly infinite), consider closed convex sets $\left\{S_{j}: j \in J\right\}$ and closed sublinear functions $\left\{\sigma_{j}: j \in J\right\}$ related by

$$
\left\{\begin{array}{l}
\sigma_{j} \text { is the support function of } S_{j} \\
\text { or equivalently: } S_{j}=\partial \sigma_{j}(0)
\end{array}\right.
$$

Form the following two pairs $(S, \sigma)$ :

\footnotetext{
${ }^{2}$ The assumption $S \neq \emptyset$ is convenient. We may also define $\sigma_{\emptyset} \equiv-\infty$, although this is a somewhat pathological "function".

${ }^{3}$ This means:

- its epigraph $\left\{(x, r) \in \mathbb{R}^{n} \times \mathbb{R}: r \geqslant \sigma_{S}(x)\right\}$ is a closed set in $\mathbb{R}^{n+1}$,

- or equivalently: its sublevel sets $\left\{d: \sigma_{S}(d) \leqslant \beta\right\}$ are closed sets (possibly empty) in $\mathbb{R}^{n}$, for any $\beta$,

- or equivalently: if $d_{k} \rightarrow d$ and $\sigma_{S}\left(d_{k}\right) \rightarrow \ell$, then $\ell \geqslant \sigma_{S}(d)$.
} 
(i) Set $S:=\overline{\operatorname{conv}}\left(\cup_{j} S_{j}\right)$ and $\sigma:=\sup _{j} \sigma_{S_{j}}$.

(ii) Set $S:=\cap_{j} S_{j}$ and let $\sigma\left[:=\overline{\operatorname{conv}}\left(\inf _{j} \sigma_{j}\right)\right]$ be the closed convex hull of the $\sigma_{j}$ 's: this is the function whose epigraph is the closed convex hull of the union of the epigraphs of the $\sigma_{j}{ }^{\prime} \mathrm{s}^{4}$

Then, in both cases (i) and (ii) we have

$$
\left\{\begin{array}{l}
\sigma \text { is the support function of } S, \\
\text { or equivalently: } S=\partial \sigma(0)
\end{array}\right.
$$

Exposed faces and normal cones Here $S$ is assumed to be closed and convex. A half-space $\left\{x: d^{\top} x \leqslant \beta\right\}$ contains $S$ if and only if $\beta \geqslant \sigma_{S}(d)$. If $\beta=\sigma_{S}(d)$ and if the supremum in (12) is attained, then the hyperplane $\left\{x: d^{\top} x=\sigma_{S}(d)\right\}$ is said to support $S$. The contact set

$$
\mathrm{F}_{S}(d):=\underset{x \in S}{\operatorname{Argmax}} d^{\top} x=\left\{x \in S: d^{\top} x=\sigma_{S}(d)\right\}
$$

is called the face (of $S$ ) exposed by $d$ (note: $\mathrm{F}_{S}(0)=S$ ).

Knowing that the normal cone to $S$ at $x \in S$ is

$$
\mathrm{N}_{S}(x):=\left\{d \in \mathbb{R}^{n}: d^{\top}\left(x^{\prime}-x\right) \leqslant 0 \text { for all } x^{\prime} \in S\right\},
$$

the following equivalence is clear:

$$
x \in \mathrm{F}_{S}(d) \quad \Longleftrightarrow \quad d \in \mathrm{N}_{S}(x) .
$$

Gauges and polar sets The polar of a set $S \subset \mathbb{R}^{n}$ is

$$
S^{\circ}:=\left\{y: y^{\top} x \leqslant 1 \text { for all } x \in S\right\}=\left\{y: \sigma_{S}(y) \leqslant 1\right\} .
$$

Being an intersection of closed half-spaces, $S^{\circ}$ is always closed and convex.

Besides (12), another function associated with $S$ is its gauge

$$
\gamma_{S}(q):=\inf _{t>0, q \in t S} t
$$

(knowing that $\gamma_{S}(q)=+\infty$ if $t q \in S$ for no $t>0$ ). Polarity establishes a duality correspondence between $\sigma$ and $\gamma$ :

$$
\text { If } S \text { is closed and convex, then } \gamma_{S}=\sigma_{S^{\circ}} \text { and } \sigma_{S}=\gamma_{S^{\circ}}
$$

(note: if $S$ is the unit-ball of some norm, (18) defines the dual norm, namely the one having $S^{\circ}$ as unit-ball).

When $S$ is a cone, (i.e. $t x \in S$ whenever $x \in S$ and $t>0$ ), $S^{\circ}$ is not changed if the righthand side "1" of (18) is replaced by any nonnegative number, say 0 . Thus, the polar cone of a cone $K$ is the closed convex cone

$$
K^{\circ}:=\left\{y: y^{\top} x \leqslant 0 \text { for all } x \in K\right\}=\left\{y: \sigma_{K}(y) \leqslant 0\right\}
$$

of directions making an obtuse angle with all directions in $K$. Note: the polar of $K$ is its normal cone at 0 .

The polar of $K^{\circ}$, i.e. the bipolar $K^{\circ \circ}$ of $K$, is just the closed convex hull of $K$ (i.e. $K^{\circ \circ}=K$ if $K$ is a closed convex cone). The gauge and support function of a closed convex cone are clearly either 0 or $+\infty$ : indeed

$$
\text { For a closed convex cone } K,\left[\gamma_{K^{\circ}}(y)=\right] \sigma_{K}(y)= \begin{cases}0 & \text { if } y \in K^{\circ} \\ +\infty & \text { otherwise }\end{cases}
$$

When $K$ is a polyhedral cone, namely $K=\left\{x \in \mathbb{R}^{n}: A x \leqslant 0\right\}$, Farkas' lemma states that $K^{\circ}=\left\{A^{\top} \lambda\right.$ : $\left.\lambda \in \mathbb{R}_{+}^{m}\right\}$.

\footnotetext{
${ }^{4}$ The epigraph of $\sigma$ is thus the intersection of the half-spaces containing the epigraphs of all the $\sigma_{j}$ 's: the property $r \geqslant \sigma(d)$ means

$$
\begin{array}{ll}
r \geqslant \alpha^{\top} d+\beta & \text { for all } \alpha, \beta \text { such that } \\
& \alpha^{\top} d^{\prime}+\beta \leqslant \sigma_{j}\left(d^{\prime}\right) \text { for all } d^{\prime} \in \mathbb{R}^{n} \text { and all } j \in J .
\end{array}
$$
}




\section{References}

[1] E. Balas. Disjunctive programming. Annals of Discrete Mathematics, 5:3-51, 1979.

[2] E. Balas, G. Cornuéjols, and S. Ceria. A lift-and-project cutting plane algorithm for mixed 0-1 programs. Mathematical Programming, 58:295-324, 1993.

[3] E. Balas and M. Perregaard. Lift-and-project for mixed 0-1 programming: recent progress. Discrete Applied Mathematics, 123:129-154, 2002.

[4] P. Bonami. Etude et mise en æuvre d'approches polyédriques pour la résolution de programmes en nombres entiers ou mixtes généraux. PhD thesis, Université de Paris 6, 2003.

[5] P. Bonami. Personal communication, 2004.

[6] S. Ceria and J. Soares. Disjunctive cuts for mixed 0-1 programming: duality and lifting. Technical report, GSB, Columbia Univ., 1997.

[7] S. Ceria and J. Soares. Convex programming for disjunctive convex optimization. Mathemtatical Programming, 86(3):595-614, 1999.

[8] W. Cook, R. Kannan, and A. Schrijver. Chvátal closures for mixed integer programs. Mathematical Programming, 47:155-174, 1990.

[9] R. Gomory. An algorithm for the mixed integer problem. Technical Report RM-2597, The Rand Coporation, 1960.

[10] J.-B. Hiriart-Urruty and C. Lemaréchal. Fundamentals of Convex Analysis. Springer Verlag, Heidelberg, 2001.

[11] P.A. Rey. Análise convexa e métodos Lift-and-Project para programação inteira. PhD thesis, Dept. of Electrical Engineering, PUC, Rio, Brazil, 2001.

[12] R.T. Rockafellar. Convex Analysis. Princeton University Press, 1970. 


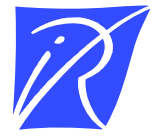

Unité de recherche INRIA Rhône-Alpes 655, avenue de l'Europe - 38330 Montbonnot-St-Martin (France)

Unité de recherche INRIA Lorraine : LORIA, Technopôle de Nancy-Brabois - Campus scientifique 615, rue du Jardin Botanique - BP 101 - 54602 Villers-lès-Nancy Cedex (France)

Unité de recherche INRIA Rennes : IRISA, Campus universitaire de Beaulieu - 35042 Rennes Cedex (France)

Unité de recherche INRIA Rocquencourt : Domaine de Voluceau - Rocquencourt - BP 105 - 78153 Le Chesnay Cedex (France)

Unité de recherche INRIA Sophia Antipolis : 2004, route des Lucioles - BP 93 - 06902 Sophia Antipolis Cedex (France)

INRIA - Domaine de Voluceau - Rocquencourt, BP 105 - 78153 Le Chesnay Cedex (France)

http://www.inria.fr

ISSN 0249-6399 